\title{
The Static Voltage Stability Research Based on the Improved Continuous Power Flow
}

\author{
An Junjun \\ School of Electrical Engineering \\ Zhengzhou University \\ Zhengzhou,China \\ e-mail: ajjijr@163.com
}

\author{
Lv Shengnan \\ State Grid Henan Electric Power Corporation \\ Maintenance Company \\ Zhengzhou,China \\ e-mail: lvshengnan1990@126.com
}

\begin{abstract}
This paper proposes an improved method of continuation power flow calculation to trace the $\mathrm{PV}$ curve of power systems .A new parametric method which is based on the algorithm principle is proposed to reduce the possibility of parametric method that does not converge in the correction process, which improves the robustness of continuous power flow. At the same time, an change-step algorithm is proposed to reduce the number of iterations of the calculation process, which improves the efficiency of the algorithm.The critical voltage and limit power of the system are tracked successfully by the proposed method with the example of IEEE39 node test system, which show the correctness and validity.
\end{abstract}

Keywords-continuation power flow ; nonlinear predictor; geometric similarity; PV curve

\section{INTRODUCTION}

Continuous Power Flow(Continuous Power Flow,CPF),also known as extension trend,is a powerful tool which based on the generator's power distribution and load changes to track the static behavior of the power system ${ }^{[1-4]}$. Continuous power flow method is to start from the stable working point of the initial load, with the load slow change, the corresponding PV curve to forecast the next working point and to correct it, until draws the outline of the complete PV curve.

PV curve due to reflect the change of the node voltage of the system with the load changing, therefore, has been widely used to determine the distance from the system point to voltage collapse point, or to determine the voltage collapse point.. The basic idea of continuous power flow method is that from the current working point, along with the load increasing, we use predictor / corrector operator to solve continuous power flow (the operating point of the system), until obtain the voltage collapse point (SNB) and the whole PV curve, at the same time, won the flow solution of the critical state of the load (voltage stability margin).

The calculation of continuous power flow in two main problems worth studying: one is that iterative calculation of the correct process is not converged, the second is that continuous power flow needs relatively large amount of calculation. To solve the above two problems, this paper proposes a new parameterized method and a change-step algorithm, thus can draw good PV curve and track the critical voltage and the power limit of the system.

In this paper,according to geometry principle to choose the parameter, the method is simple, easy to understand,and also has better convergence. At the same time,the method uses the change-step algorithm to correct, which improves the efficiency of program. The critical voltage and limit power of the system are tracked successfully by the method with the example of IEEE39 node test system, which show the correctness and validity.

\section{THE MODEL OF CONTINUOUS POWER FLOW}

Conventional power flow equation can be written as:

$$
\left\{\begin{array}{c}
0=P_{G i}-P_{L i}-\sum_{j=1}^{n} V_{i} V_{j}\left(G_{i j} \cos \theta_{i j}+B_{i j} \sin \theta_{i j}\right) \\
0=Q_{G i}-Q_{L i}-\sum_{j=1}^{n} V_{i} V_{j}\left(G_{i j} \sin \theta_{i j}-B_{i j} \cos \theta_{i j}\right) \\
(i=1, \cdots, n)
\end{array}\right.
$$

Among them: $P_{G i} \quad Q_{G i}$ are the output of the node generator; $P_{L i} Q_{L i}$ are the node load; $V_{i} \theta_{i}$ are the node voltage magnitude and phase of the node $i ; G_{i j} B_{i j}$ are the real and imaginary part of the admittance matrix of the element $(i, j)$.

Based on the idea of continuation method, introducing the parameter $\lambda$ to reflect the change of $P_{G i} Q_{G i}$ and $P_{L i}$ $Q_{L i}$ ( $\lambda$ also to reflect the change of $G_{i j} B_{i j}$ ), parametric power flow equation can be written as:

$$
\begin{aligned}
& \left\{\begin{array}{l}
0=\left(1+\lambda K_{G P i}\right) P_{G i}-\left(1+\lambda K_{L P i}\right) P_{L i}-\sum_{j=1}^{n} V_{i} V_{j}\left(G_{i j} \cos \theta_{i j}+B_{i j} \sin \theta_{i j}\right) \\
0=\left(1+\lambda K_{G Q i}\right) Q_{G i}-\left(1+\lambda K_{L Q i}\right) Q_{L i}-\sum_{j=1}^{n} V_{i} V_{j}\left(G_{i j} \sin \theta_{i j}-B_{i j} \cos \theta_{i j}\right) \\
\left(i=1, \cdots, n ; \lambda \in\left[0, \lambda_{c r}\right]\right)
\end{array}\right. \\
& \left(K_{G P i} \in[0,1] ; K_{L P i} \in[0,1] ; K_{G Q i} \in[0,1] ; K_{L Q i} \in[0,1]\right)
\end{aligned}
$$

Among them, $K_{G P i} K_{L P i} K_{G Q i} K_{L Q i}$ are the growth ratio coefficient of continuous power flow, and can be set according to the actual situation. The equation can be simplified as:

$$
f(\bar{x}, \bar{y}, \bar{z}, \lambda)=\overline{0}
$$

Among them, $f(\cdot)$ are the nonlinear power flow equations; $\bar{x}$ is the vector of $V_{i} \theta_{i} ; \bar{y}$ is the vector of $P_{G i}$ 
$Q_{G i}$ and $P_{L i} Q_{L i} ; \bar{z}$ is the vector of $G_{i j} B_{i j} ; \lambda$ reflects the change way of $\bar{y} \quad \bar{z}$. The solutions of flow equation depends on the parameter changes, and their relationship in a multidimensional parameter space maps out a solution manifold.

\section{THE ALGORITHM ANALYSIS AND IMPROVEMENT OF CONTINUOUS POWER FLOW}

Continuous power flow from established steady equilibrium point on a curve, according to the specified parametric way, through the predictor corrector tracking solution manifold, until gets a critical point. Continuous power flow calculation is made up of four parts: 1) the parameterization; 2) predict steps; 3) correction steps; 4) step length control.

\section{A. Improved parameterization}

Parameterization is the core of a continuous power flow, which runs through prediction and correction links. Due to the introduction of the parameter,the number of unknowns are more than the number of equations, which result the equations can not be solved directly. For determining the solution must be added in an equation, then solve the equation and the original equations to get a solution, which is the essence of parameterized. If in the prediction process the tangent prediction method is applied to calculate the equations, then the correction process also requires the application of parametric methods. Both are mutually independent with different forms, and also have some connection. At present there are mainly local parameterized method and global parameterization method. Literature [6] proposed local parameterized using a single continuous parameter. Literature [4] proposes a continuous arc length method. [5] proposed a quasi arc length method.

This paper proposes the improved parameterization method which is based on local parameterized geometric similar method. As shown in figure 1 , point $A$ is the predicted values $\left(x_{k}^{c}, \lambda^{c}\right)$ of the $\mathrm{k}$-th state variable, point $B$ is the solution of the corresponding continuous power flow, $C D$ is respectively the projection on the load factor $\lambda$ shaft. In figure 1 , it is easy to find the triangle $A O C$ is similar to the triangle $B O D$, and exist $\frac{A C}{O C}=\frac{B D}{O D}$. Choosing a starting point $\left(x_{k}^{0}, \lambda^{0}\right)$ on the surface, then it can obtain the prediction point $\left(x_{k}^{c}, \lambda^{c}\right)$.The origin usually is $O(0,0)$, and at this time there are:

$$
\frac{x_{k}^{c}-x_{k}^{0}}{\lambda^{c}-\lambda^{0}}=\frac{x_{k}-x_{k}^{0}}{\lambda-\lambda^{0}}
$$

That is

$$
G(\theta, U, \lambda)=\left(x_{k}-x_{k}^{0}\right)-\alpha\left(\lambda-\lambda^{0}\right)=0
$$

Among them, $\alpha=\frac{x_{k}^{c}-x_{k}^{0}}{\lambda^{c}-\lambda^{0}}$ is named for the similar ratio factor. Once the predicted value is determined, the similar ratio factor is determined.Selection principle of the continuous parameter is that at first,the voltage change of two continuous points of the PV curve divided by the corresponding voltage amplitude, and then take the parameter who has the largest absolute value of the corresponding state variables as continuous parameters, such as the expression (6):

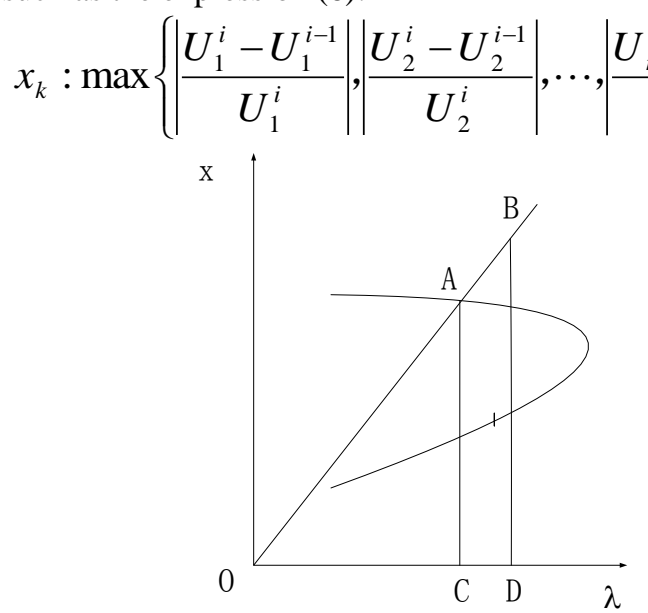

Figure 1. the parameterized geometric figure

By Newton method for solving the equations (3) and (5) can get an flow solution,at the same time,can get a new expansion power flow equations, such as equation (7).

$$
\left[\begin{array}{cc}
J & H_{\lambda} \\
e_{k} & -\alpha
\end{array}\right]\left[\begin{array}{l}
d x \\
d \lambda
\end{array}\right]=\left[\begin{array}{c}
\Delta H \\
\Delta G
\end{array}\right]
$$

Among them, $J$ is the Jacobi matrix of conventional flow equation; $H_{\lambda}$ is the load factor derivative of conventional flow equation; $e_{k}$ is a row vector, in addition to the corresponding parameter recurrently elements is equal to 1 , the other elements are equal to zero.

\section{B. Predicting}

Predicting is an important part of continuous power flow, predicted results is directly related to the robustness of continuous power flow and computational efficiency. The purpose of continuous power flow predicting link is to find out the approximate solution of the next solution, and provide initial values for Newton or quasi-Newton iterative calculation in correction steps. The common method to predict in general can be divided into two categories, linear and nonlinear prediction. Tangent linear prediction method is used more often. Literature[7] introduces the application of the tangent method. Literature[8] introduced the application of secant method. The accuracy of tangent method can meet the needs of most projects, and the tangent method often need to calculate the tangent vector to determine whether continuous flow through the critical point.For the tangent method, the state variables and parameters of differential of the calculation formula(3) is needed, namely:

$$
d f=\frac{\partial f}{\partial \bar{x}} d \bar{x}+\frac{\partial f}{\partial \lambda} d \lambda=\overline{0}
$$

the calculation formula(4) can also be written as: 


$$
[J]\left[\begin{array}{l}
d \bar{x} \\
d \lambda
\end{array}\right]=\left[\begin{array}{ll}
\frac{\partial f}{\partial \bar{x}} & \frac{\partial f}{\partial \lambda}
\end{array}\right]\left[\begin{array}{l}
d \bar{x} \\
d \lambda
\end{array}\right]=\overline{0}
$$

Among them, $J$ is the corresponding Jacobi matrix of parameterized flow equations. Apparently the formula (8) is described by a straight line in a multidimensional space. Local parameterization, orthogonal parameterized and quasi arc length parametric method is to construct a hyperplane. The arc length parametric method is to construct a hypersphere. It is noted that the latter need to use special methods which can bring extra amount of calculation, so from the perspective of improving the efficiency, the former is superior to the latter. Prediction has two basic principles: one is the proximity of the solution, namely the predicted value need to fully close to the manifold of the continuous power flow. Second is the directional, namely the direction of $\left(d \overline{x_{0}}, d \lambda_{0}\right)$ must ensure that the continuous power flow along a fixed direction tracks solutions of the manifold ${ }^{[9]}$.To construct a hyperplane, its parameters is derived from the solution of the previous prediction process. In order to ensure the approximation of the predictive value, $\left(d \overline{x_{0}}, d \lambda_{0}\right)$ can be converted into a unit. In order to ensure the direction of the predicted process, you can use the method of literature [10], with the unit tangent vector inner product between two steps reflecting the tracking direction of the continuation method (If the inner product is positive, then the direction is the same; If the inner product is negative, then the direction is the different.).

The predicted value is:

$$
\left\{\begin{array}{l}
(\bar{x}, \lambda)=\left(\overline{x_{0}}, \lambda_{0}\right)+\sigma \frac{\left(d \overline{x_{0}}, d \lambda_{0}\right)}{\left\|d \overline{x_{0}}, d \lambda_{0}\right\|}\left(\left(d \overline{x_{0}}, d \lambda_{0}^{\prime}\right)\left(d \overline{x_{0}}, d \lambda_{0}\right)>0\right) \\
(\bar{x}, \lambda)=\left(\overline{x_{0}}, \lambda_{0}\right)-\sigma \frac{\left(d \overline{x_{0}}, d \lambda_{0}\right)}{\left\|d \overline{x_{0}}, d \lambda_{0}\right\|}\left(\left(d \overline{x_{0}^{\prime}}, d \lambda_{0}^{\prime}\right)\left(d \overline{x_{0}}, d \lambda_{0}\right)<0\right)
\end{array}\right.
$$

Among them: $(\bar{x}, \lambda)$ is the predicted value; $\left(\overline{x_{0}}, \lambda_{0}\right)$ is the basic value; $\sigma$ is the step control factor, $\left(d \overline{x_{0}^{\prime}}, d \lambda_{0}^{\prime}\right)\left(d \overline{x_{0}}, d \lambda_{0}\right)$ is respectively the predicted tangent vector of the previous step and this step. According to the above improvements, the method can not only ensure the approximation of the predicted process, but also take the direction into account.

\section{The improvement of the change-step algorithm}

To control the step is for the control of the step length control factor of the formula(10), thus essentially is the control of the predicted value of the continuous power flow. For the effective control of the predicted value,making the tangent vector of the predicted value convert into a unit is indispensable. The presented algorithm in the paper, is also processing on the basis of the unit of the predicted vector.

Step change includes two aspects: one is the growth of the step, the other is the decrease of the step. When the number of iterations is smaller than a certain value in the predicted process ,the corresponding step length increases, and when the number of iterations in the predicted process exceeds a certain value, the step length decreases.
The computation formula of the step length parameter is as follows:

When $L_{n} \leq 1$, then $\sigma_{n+1}=\beta \sigma_{n}$;

When $L_{\max }>L_{n}>1$, then $\sigma_{n+1}=\alpha^{L_{n-1}} \sigma_{n}$;

When $L_{n}>L_{\max }$, then $\sigma_{n+1}=\alpha^{L_{n-1}} \sigma_{n}$, back to calculate.

Among them $(0<\alpha<1,0<\beta<1)$.

\section{Correcting}

After a predicted value is obtained by the predicted process, for obtaining the actual flow values the error correction is needed.In this paper using parametric method introduced by 2.1 predict the predicted value.In principle, all the valid values for solving nonlinear algebraic equations can be to correct the error, and the predicted value of the continue power flow and actual value of that is the closer,the fewer number of corrections.

\section{ANALYSIS OF EXAMPLES}

In the article examples use local parametric methods in predicted process,and the predicted vector is normalized;correction process is based on the geometrical similarity of local parameterization. In this paper,an example of IEEE39 node system are calculated and analyzed; the system does not consider the generator reactive power limit; all loads and the active and reactive power of generators are growth in the same proportion $\lambda$; the load model is constant power model, and maintain that the power factor is a constant.Taking $\varepsilon=10^{-5}$,among them $\varepsilon$ is the required precision of the node power deviation.In step length formula we take $\sigma^{1}=0.1, \alpha=0.5, \beta=0.5$.

Figure 2 is the $\lambda V$ curve of the node 11 in IEEE39 node system.Using the method described in this article effectively avoids that the Jacobi matrix is singular in power limit place, and obtain the critical voltage value and limit power value. Table 1 shows the datas of the $\lambda V$ curve of node 11 . As can be seen from the table, when all loads are growth,the load growth factor of IEEE39 node system is $\lambda=1.2722$, reaching the inflexion point of the $\lambda V$ curve, at the moment,the voltage of the corresponding node 11 is critical voltage value $U_{c}=0.7075$, then loads begin to fall, and the voltage continue to low,that is the second half of the curve. As can be seen from the number of iterations, even near the critical point, the number of iterations which can convergence are just four times.

In Figure 2 the whole network simulation results of the curve of IEEE39 node system and the results of the literature [11] are basically the same, indicating the validity of the proposed method. 


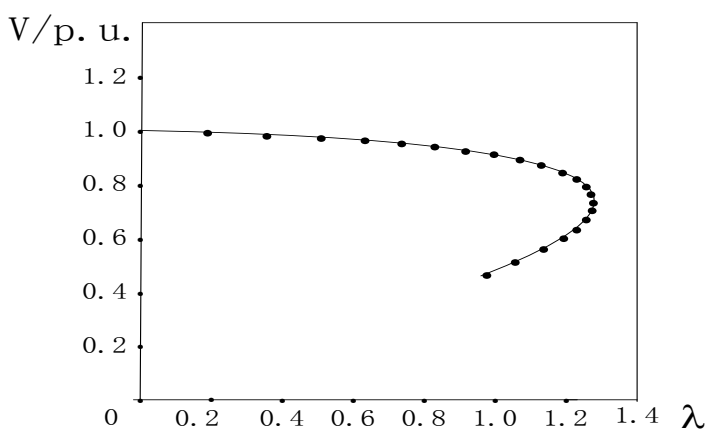

Figure 2. the $\lambda V$ curve of the node 11 in IEEE39 node system

TABLE I. THE DATAS OF THE $\lambda V$ CURVE OF NODE 11 IN IEEE39 NODE SYSTEM

\begin{tabular}{|ccc|ccc|}
\hline $\begin{array}{c}\text { load factor } \lambda \\
\text { iterations }\end{array}$ & \multicolumn{3}{|c|}{$\begin{array}{c}\text { load factor } \lambda V_{11} / p . u . \\
\text { iterations }\end{array}$} \\
\hline 0.0 & 1.0224 & 3 & 1.2143 & 0.7963 & 4 \\
0.2073 & 1.0029 & 3 & 1.2444 & 0.7733 & 4 \\
0.3656 & 0.9856 & 3 & 1.2633 & 0.7512 & 4 \\
0.5132 & 0.9677 & 3 & 1.2723 & 0.7291 & 4 \\
0.6450 & 0.9490 & 3 & 1.2729 & 0.7075 & 4 \\
0.7676 & 0.9286 & 3 & 1.2653 & 0.6868 & 4 \\
0.8745 & 0.9079 & 3 & 1.2503 & 0.6666 & 4 \\
0.9693 & 0.8787 & 3 & 1.2293 & 0.6462 & 4 \\
1.0501 & 0.8643 & 3 & 1.2017 & 0.6273 & 4 \\
1.1182 & 0.8419 & 3 & 1.1682 & 0.6084 & 4 \\
1.1726 & 0.8192 & 3 & 1.1273 & 0.5903 & 3 \\
\hline
\end{tabular}

\section{THE CONCLUSION}

This paper proposes a new parametric method of continuous power flow, through drawing PV curve, to track the critical voltage and limit power of the system. The method solves the problem that the Jacobi matrix of the general flow equations is singular in the extreme power. The method is simple, and easy to understand and has better convergence and higher efficiency. The method of IEEE39 node test system carried on the example analysis, has obtained the ideal effect, so as to show the correctness and validity of the method.

\section{REFERENCES}

[1] Wang Xifan,Fang Wanliang,Du Zhengchun.Modern power system analysis[M].Science Press,2003.

[2] Ajjarapu V,Christy C.The continuation power flow:a tool for steady state voltage stability analysis[J].IEEE Trans. on Power Systems, 1992,7(1):416-423.

[3] Canizares C A,Alvarado F L. Point of collapse and continuation methods for large ac/dc system[J].IEEE Trans. on Power System,1993,8(1):1-8.

[4] Chiang H D,Flueck A J,Shah K. S,Balu N.CPFLOW.A practical tool for tracing power system steady-state stationary behavior due to load and generation variations[J].IEEE Trans. on Power Systems, 1995,10(2):623-634.

[5] Flueck A J,Dondeti J R.A new continuation power flow tool for investigating the nonlinear effects of transmisson branch parameter variations[J].IEEE Trans on Power System,2000,15(1):223-227.

[6] Li Lin.An improved parameterization of continuous power flow calculation method[J]. Modern Electric Power, 2011, 28 (5) : 10-14.

[7] Du Xiaoming,Zhao Dongmei. Improved method of continuous flow to trace the PV curve[J]. Power System Technology,2006,30:278-281.

[8] Wang Hongyan,Cai Liang,Qi Zhongjian. An improved continuous flow algorithm to calculate the PV curves of power system[J]. electrical technology,2011,7:28-31.

[9] Dong Xiaoming,Han Xueshan.Continuous flow analysis and practical improvements taking the error factors into account[A]. Power system planning and the analysis and control of homeostasis, 219-222.

[10] Li Qingyang. Numerical solution of nonlinear equations[M]. Science Press, 1987.

[11] Peng Zhiwei,Hu Guogen,Han Zhenxiang. Voltage stability analysis of power system based on bifurcation theory[M].China Electric Power Press, 2005 